\title{
Affine Formation Algorithms and Implementation Based on Triple-Integrator Dynamics
}

DOI:

10.1142/S2301385019400041

\section{Document Version}

Accepted author manuscript

Link to publication record in Manchester Research Explorer

\section{Citation for published version (APA):}

Onuoha, O., Tnunay, H., Li, Z., \& Ding, Z. (2019). Affine Formation Algorithms and Implementation Based on Triple-Integrator Dynamics. Unmanned Systems, 7(1), 33-45. https://doi.org/10.1142/S2301385019400041

\section{Published in:}

Unmanned Systems

\section{Citing this paper}

Please note that where the full-text provided on Manchester Research Explorer is the Author Accepted Manuscript or Proof version this may differ from the final Published version. If citing, it is advised that you check and use the publisher's definitive version.

\section{General rights}

Copyright and moral rights for the publications made accessible in the Research Explorer are retained by the authors and/or other copyright owners and it is a condition of accessing publications that users recognise and abide by the legal requirements associated with these rights.

\section{Takedown policy}

If you believe that this document breaches copyright please refer to the University of Manchester's Takedown Procedures [http://man.ac.uk/04Y6Bo] or contact uml.scholarlycommunications@manchester.ac.uk providing relevant details, so we can investigate your claim.

\section{OPEN ACCESS}




\title{
Affine Formation Algorithms and Implementation Based on Triple-Integrator Dynamics ${ }^{*} \dagger$
}

\author{
Okechi Onuoha, Hilton Tnunay, Zhenhong Li, Zhengtao Ding \\ School of Electrical amd Electronic Engineering, University of Manchester, Manchester, M13 9PL, UK. \\ E-mail: okechi.onuoha@manchester.ac.uk, hilton.tnunay@manchester.ac.uk \\ Zhenghong.liemanchester.ac.uk, Zhengtao.Dingemanchester.ac.uk
}

This paper presents novel affine formation algorithms and implementations in different scenarios for the coordination of multi-agent systems with triple-integrator agent dynamics for both sampled-data and continuous-time settings. The agents in affine maneuver control are to be capable of producing required geometric shapes and simultaneously accomplishing desired maneuvers such as shearing, rotation, translation and scaling. From existing work, these tasks can be accomplished for systems whose agent dynamics are described using double-integrators and the agents communicate continuously in time. In some practical situations, however, the inter-agent communication may be limited to periodic intervals of time. Furthermore, a wide range of systems is governed by complex dynamics described with higher-orders. This paper presents two novel algorithms based on triple-integrator agent dynamics. Four implementation cases comprising of two scenarios each studied in both continuous-time and sampled-data cases are considered. Under the proposed algorithms, the collection of agents are capable of tracking time-varying targets which are affine transforms of the reference formation, if the leaders have knowledge of the required formation maneuvers. Detailed implementation results are presented to demonstrate the efficacy of the proposed algorithms.

Keywords: Formation Control; Multi-Agent System; Cooperative Control; Autonomous Systems; Multi-Agent Coordination; Stress Matrix; Affine Formation Control.

\section{INTRODUCTION}

The multi-agent system (MAS) formation control problem has attracted increased attention from researchers in recent times because of its application to a wide range of areas. This includes the control of unmanned ariel vehicles, satellite clusters, coordination of teams of mobile robots, and so on. The ultimate goal of formation control is to design distributed control laws that ensure that the agents of a given MAS both form desired geometric patterns and collectively achieve any required maneuver.

Different strategies have been proposed to address the formation control problem of MASs. For example, the consensusbased strategy is used to address the formation control problem in $[1,2]$.This strategy is grouped into three approaches by convention. They are the bearing-based [3], displacementbased [4] and distance-based [5] approaches. These approaches achieve the defined formation by defining constant constraints on the inter-agent bearing, displacement and distance. These preset constant offsets in turn negatively impose limitations on the maneuvers the formation, as a whole, can carry out. For in- stance, consensus-based formation control laws based on the displacement approach can track target functions having timevarying translations $[6,7]$ but carrying out scales on the formation would require a redesign of the displacements. Similarly, formation control laws based on distance can track formation targets with time-varying translations and orientations [8, 9], but have difficulties in tracking target formations having timevarying scales. Also, the bearing-based formation control laws can track time-varying formation translations and scales [5, 3], but have difficulties in tracking time-varying formation orientations. Thus, simultaneously achieving translation, rotation and scaling maneuvers by any of these methods are involving. The Complex Laplacian-based strategy $[10,11]$, has been proposed to extend the maneuverability of formation of MASs. This strategy is able to carry out maneuvers such as rotation, translation and scaling. Unfortunately, the strategy is only able to address two-dimensional systems. This inadequacy motivated further research which led to the development of the strategy based on the stress matrix.

The prospect of the stress matrix based strategy is good

\footnotetext{
*This paper is a comprehensive version of our conference paper, "Affine Formation Maneuver Control of Multi-Agent Systems with TripleIntegrator Dynamics," submitted for American Control Conference 2019.

${ }^{\dagger}$ This work was supported in part by the Petroleum Technology Development Fund (PTDF).
} 
with the promise of being able to achieve general formation maneuvers in all dimensions. Both Laplacian and stress matrices have similar properties. However, the respective edge entries producing the stress matrix need to be jointly determined by the formation configuration. Furthermore, the respective values of the weight (edge) entries of the stress matrix can be either positive or negative.

Recently, the formation control strategy based on the stress matrix has been applied to the affine formations control of MASs. An affine transformation is viewed as a general linear transformation corresponding to translation, rotation, shearing, scaling, or any combination of them. The affine formation control problem based on the stress matrix with stationary leaders is studied for agent dynamics described by singleintegrator in [12]. Formation scaling is considered in [13]. The affine formation control problem where the leaders are dynamic or modelled with double-integrator agent dynamics is addressed in [14]. These studies only considered the case where inter-agent communication (or sensing) occur continuously in time and the agents' dynamics are limited to double-integrators.

In real-life situations, however, agents may only communicate in periodic time intervals and may have agent dynamics described by triple-integrators. Triple-integrator agent dynamics have found applications in robot motion control, aircraft control, lifts and a wide range of mechanical control systems. For example, the DC motor which features as an actuator in a vast majority of mechanical control systems is normally modelled with triple-integrator agent dynamics when the motor load is considered. Triple-integrator agent dynamics are used to approximate the individual agent dynamics in an $n$-vehicle system of travelling along a single lane for a drive-train model in [15]. A broad range of systems could be modelled using triple-integrator agent dynamics, see $[16,17]$. Therefore, it is important to broaden the application area by considering the stability conditions for MASs with triple-integrator agent dynamics.

In this paper, we study the affine formation maneuver control problem of MASs described by triple-integrator agent dynamics. We propose two control laws based on periodic and continuous communications. Sufficient conditions are presented to guarantee the global stability of the proposed control laws. The proposed laws are implemented for four cases consisting of two scenarios each considered for both sampled-data and continuous-time agent communication cases.

The remainder of this paper is organised as follows. Preliminary results and notations used in the remainder of this paper are presented in Section 2. The affine formation control problem is formulated in Section 3. Section 4 considers the continuous-time case of the affine formation maneuver control problem. Section 5 studies the sample-data case. Section 6 presents simulation study of the problem. Finally, a conclusion for the study is presented in Section 7.

\section{PRELIMINARIES}

This section presents some preliminary results and notations used in the remaining sections of this paper.

\subsection{Basic Graph Notations}

Consider a MAS composed of $n$ agents. Let the communication among the agents be modelled by a graph $\mathscr{G}(\mathscr{V}, \mathscr{E})$, which denotes a collection of edges $\mathscr{E}$ and vertices $\mathscr{V}$. An edge denotes a direct communication path from one node (or vertice) to another. For example, if node $j$ can obtain information from node $i$ (or node $i$ can transmit information to node $j$ ), then edge $(i, j)$ is considered to exist, i.e., $(i, j) \in \mathscr{E}$. The edge $(i, j)$ has a weight given by $w_{j i}$ if it exists and 0 if it does not. If for every existing edge $(j, i) \in \mathscr{E}$; we have $(i, j) \in \mathscr{E}$, then the graph is said to be undirected, but if otherwise, the graph is considered directed. The neighbours of node $i$ are the set of nodes it can receive information from. This set is denoted by $\mathscr{N}_{i}$. In this paper, we assume each agent knows its state and the states of its neighbours. Also, $\otimes$ denotes the Kronecker product.

A configuration denotes a collection of nodes defined by their respective positions in Euclidian coordinate space, $\mathbb{R}^{d}$. We denote this by $p=\left[p_{1}^{T}, \ldots, p_{n}^{T}\right]^{T}$, where $p_{i} \in \mathbb{R}^{d}$. A framework $\mathscr{F}=(\mathscr{G}, p)$ in $\mathbb{R}^{d}$ is a graph defined with its configuration. Two frameworks, $(\mathscr{G}, q)$ and $(\mathscr{G}, p)$ are considered to be equivalent, written as $((\mathscr{G}, q) \equiv \mathscr{G}, p)$, if and only if

$$
\left\|q_{i}-q_{j}\right\|=\left\|p_{i}-p_{j}\right\|, \quad \forall(i, j) \in \mathscr{E} .
$$

The two frameworks, $(\mathscr{G}, q)$ and $(\mathscr{G}, p)$, are considered to be congruent, written as $(\mathscr{G}, p) \cong(\mathscr{G}, q)$, if and only if

$$
\left\|q_{i}-q_{j}\right\|=\left\|p_{i}-p_{j}\right\|, \quad \forall i, j \in \mathscr{V} .
$$

A framework in $\mathbb{R}^{d}$ is considered globally rigid if $(\mathscr{G}, p) \equiv$ $(\mathscr{G}, q)$ implies $(\mathscr{G}, p) \cong(\mathscr{G}, q)$. This follows that, any framework in $\mathbb{R}^{d}$ equivalent to $(\mathscr{G}, p)$ is congruent to it as well. A configuration $p$ is considered universally rigid if and only if for all $\mathbb{R}^{d_{1}}$, where $d_{1}$ refers to any positive integer, $(\mathscr{G}, p) \equiv(\mathscr{G}, q)$ implies $(\mathscr{G}, p) \cong(\mathscr{G}, q)$. This means that, universal rigidity confers global rigidity but global rigidity does not automatically implies universal rigidity [18, 19 and 12].

\subsection{Affine Span}

The affine span, $\mathscr{S}$, of a set of points, $\left\{p_{i}\right\}_{i=1}^{n} \in \mathbb{R}^{d}$ is described by

$$
\mathscr{S}=\left\{\sum_{i=1}^{n} a_{i} p_{i}: a_{i} \in \mathbb{R} \forall i \text { and } \sum_{i=1}^{n} a_{i}=1\right\} .
$$

A collection of $d+1$ affinely independent points are required to affinely span any $d$-dimensional space. The affine span of any two distinct points is a line that joins both of them. Similarly, the affine span of three unique points that are not collinear is a 2-dimensional plane passing through these three points. Other higher dimensions follow the analogy.

Any given affine span can be easily translated so that it contains the origin, and therefore, a linear space with the same dimension as the affine space. Therefore, given a $d$-dimensional 
affine span, one can say the points span $\mathbb{R}^{d}$ affinely. In this paper, the $d+1$ leaders that span $\mathbb{R}^{d}$ is considered for the affine formation control of a $d$ dimensional space.

\subsection{Stress Matrix}

Given a framework, $\mathscr{F}=(\mathscr{G}, p)$ whose graph is undirected, we refer to the stress as a collection of the weights, $w_{i j}$ of respective edges. A stress satisfying

$$
-\sum w_{i j}\left(p_{i}-p_{j}\right)=0, \quad i \in \mathscr{V}
$$

is said to be an equilibrium stress [18, 19]. Equation (1) is written in a matrix form as

$$
-\left(\Omega \otimes I_{d}\right) p=0
$$

where the stress matrix, $\Omega \in \mathbb{R}^{n \times n}$, is given by

$$
\Omega_{i j}= \begin{cases}\sum_{j \in \mathscr{N}_{i}} w_{i j(k)}, & \text { for } i=j, \\ -w_{i j}, & \text { for } i \neq j, \quad(j, i) \in \mathscr{E}, \\ 0, & \text { for } i \neq j, \quad(j, i) \notin \mathscr{E} .\end{cases}
$$

Both classical Laplacian matrices and stress matrices of graphs have similar properties, but that the off-diagonal entries of stress matrices can be positive, negative or zero, unlike the Laplacian matrices which only permit zero and negative values. The stress matrix $\Omega$ is normally partitioned, for convenience, as

$$
\Omega=\left[\begin{array}{ll}
\Omega_{l l} & \Omega_{l f} \\
\Omega_{f l} & \Omega_{f f}
\end{array}\right],
$$

where $\Omega_{f f}$ and $\Omega_{l l}$ are respectively $n_{f} \times n_{f}$ and $n_{l} \times n_{l}$ submatrices. Note that the value of every $w_{i j}$ is to be computed.

\subsection{Stress Matrix Design}

Rigidity plays an important role in stress-matrix based formation control. The uniqueness of a framework in the entire space of a defined dimension is guaranteed by its global rigidity. Additionally, universal rigidity is required to guarantee the uniqueness of the framework in all dimensions. Unfortunately, the necessary and sufficient conditions to guarantee global/universal rigidity is still lacking in existing literature. Thus, researchers mostly concerned with MAS coordination typically focus on the special global and universal rigidity cases where the framework has a generic configuration. A configuration is considered generic if its coordinates are algebraically independent over the integer [18]. For frameworks with generic configurations, sufficient conditions are given in [18, 20 and 21] to guarantee global/universal rigidity. In the rest of this paper, we assume that our configurations are generic.

Consider a framework whose communication graph is undirected, universally rigid and has a generic configuration. Assume that $q_{1}, \ldots, q_{d}$ denote the 1 st $, \ldots, q$ th components of the configuration with $d$-dimensional nodes. For instance, consider a 3-dimensional configuration $(x, y$ and $z)$ of three unique nodes, $p_{1}(1,0,3), p_{2}(2,1,1)$ and $p_{3}(1,0,0)$, i.e., $p_{i}(x, y, z)$. This follows that $q_{1}=[1,2,1]^{T}, q_{2}=[0,1,0]^{T}$ and $q_{3}=[3,1,0]^{T}$. Note that $1_{n}, q_{1}, . ., q_{d}$ are linearly independent since the configuration is generic.

Lemma 2.1. [20, 18, 21]: A framework $(\mathscr{G}, p)$ whose graph is undirected and has a generic configuration in $\mathbb{R}^{d}$ with $n \geq d+2$ nodes is universally rigid if and only if its communication graph is $(d+1)$-connected with a stress matrix, $\Omega$, that is positive semi-definite and has a rank of $n-d-1$.

Assumption 1. The framework $(\mathscr{G}, p)$ is assumed to be generically universally rigid.

Remark 2.2. Assumption 1 guarantees that the $\operatorname{rank}(\Omega)$ of the stress matrix is $n-d-1$.

A method of computing the stress matrix as given in [14] is presented. Denote $w$ with the stress vector of the reference formation. Let the graph have $m$ undirected edges. Choose any orientation for the graph and let the incidence matrix be given by $H \in \mathbb{R}^{m \times n}$. Let $h_{i} \in \mathbb{R}^{m}$ denote the $i$ th column of the incidence matrix $H$, so that $H=\left[h_{1}, \ldots, h_{n}\right]$. Choose

$$
Z=\left[\begin{array}{c}
\bar{P}^{T}(r) H^{T} \operatorname{diag}\left(h_{1}\right) \\
\vdots \\
\bar{P}^{T}(r) H^{T} \operatorname{diag}\left(h_{1}\right)
\end{array}\right] \in \mathbb{R}^{n(d+1) \times m},
$$

where $\bar{P}(r)$ denotes the matrix $\left[q_{1}, . ., q_{d}, 1_{n}\right]^{T}$. Denote the basis of the null space of $Z$, $\operatorname{null}(Z)$ by $z_{1}, \ldots, z_{n} \in \mathbb{R}^{m}$. Let the singular value decomposition (SVD) of $\bar{P}(r)=U \sum V$. Let $U=\left[\begin{array}{ll}U_{1} & U_{2}\end{array}\right]$, where the first $d+1$ columns of $U$ is used to compose $U_{1}$. By defining

$$
M_{i}=U_{2}^{T} H^{T} \operatorname{diag}\left(z_{i}\right) H U_{2}, \quad \forall i=1, . ., q
$$

and choosing $c_{1}, \ldots, c_{q}$ such that,

$$
c_{i} M_{i} \succ 0,
$$

the stress vector is given by

$$
w=\sum_{i=1}^{q} c_{i} z_{i} .
$$

More details on this method is contained in [14]. Furthermore, some useful guideline on constructing universally rigid frameworks is available in [22].

\subsection{Affine Realizability and Leaders Selection}

Proper leader selection is important for the manipulation of the entire formation. This subsection presents a guide on how to select the leaders for the required affine formation maneuver control.

Lemma 2.3. [14] Consider that the nominal formation of the framework $(\mathscr{G}, p)$ is composed of $n_{f}$ followers and $n_{l}$ leaders. Then, the followers target positions $p_{f}^{*}$ can be uniquely calculated from the relation

$$
p_{f}^{*}=-\left(\Omega_{f f}^{-1} \Omega_{f l} \otimes I_{d}\right) p_{l}^{*}
$$


for any $p=\left[p_{l}^{T}, p_{f}^{T}\right]^{T}$ which belongs to the collection of affine transform of the reference (nominal) formation, if and only if the set of leaders chosen span $\mathbb{R}^{d}$ affinely and $\Omega_{f f}$ is nonsingular.

Note that $p_{l}$ and $p_{f}$ respectively denote the positions of the leaders and followers while $p_{l}^{*}$ and $p_{f}^{*}$ respectively denote the target positions of the leaders and followers. Assume that the tracking error be defined by

$$
\delta_{p_{f}}(t)=p_{f}(t)-p_{f}^{*}(t)=p_{f}(t)+\left(\Omega_{f f}^{-1} \Omega_{f l} \otimes I_{d}\right) p_{l}^{*}(t),
$$

The following assumption is to guarantee that the leaders are properly chosen and the targets of the followers are defined.

Assumption 2. Assume that the $d+1$ leaders are selected such that they span the $\mathbb{R}^{d}$ space affinely.

\section{Problem Formulation}

Consider a MAS with $n$ agents. Let the position of the $i$ th agent be denoted by $x_{1}, \ldots, x_{n} \in \mathbb{R}^{d}$, so that the $i$ th agent's target position in the time-varying formation is given by

$$
x_{i}^{*}(t)=A(t) r_{i}+b(t)
$$

where both $b(t) \in \mathbb{R}^{d}$ and $A(t) \in \mathbb{R}^{d \times d}$ are time-varying and the nominal (constant reference) configuration is denoted by $r_{i} \in \mathbb{R}^{d}$. Equation (5) is written in global form as

$$
x^{*}(t)=\left[I_{n} \otimes A(t)\right] r+\mathbf{1}_{n} b(t)
$$

where $r=\left[r_{1}^{T}, \ldots, r_{n}^{T}\right] \in \mathbb{R}^{n d}$ and $x^{*}(t) \in \mathbb{R}^{n d}$ respectively denote the reference configuration and the targets (time-varying) to be tracked. The affine image is the set of all affine transform of the nominal configuration. Note that the tracked time-varying targets are affine images of the nominal configuration $r$.

We define the affine image as a collection of all the affine transformation of the reference configuration $r$. Note that the time-varying targets are affine images of the reference configuration. The affine image is given in global form by [12]

$\mathscr{A}(r)=\left\{x \in \mathbb{R}^{d n}: x=\left(I_{n} \otimes A\right) r+1_{n} \otimes b, A \in \mathbb{R}^{d \times d}, b \in \mathbb{R}^{d}\right\}$.

The overall goal is to find conditions that guarantees that

$$
\lim _{x \rightarrow \infty} x(t)=x^{*}(t), \forall x^{*}(t) \in \mathscr{A}(r) .
$$

\section{Affine Formation Control Law for Continuous-time Coordination of Multi-agent Systems Described by Triple-Integrator Agent Dynamics}

Consider the MAS where the agents communicate continuously in time, their inter-agent communication is modelled using an undirected graph, and each agent has dynamics described using triple-integrator, such that

$$
\left\{\begin{aligned}
\dot{x}_{i(t)}= & v_{i(t)}, \\
\dot{v}_{i(t)}= & a_{i(t)}, \\
\dot{a}_{i(t)=} & -\sum_{j \in \mathscr{N}_{i}} w_{i j}\left[k_{x}\left(x_{i(t)}-x_{j(t)}\right)+k_{v}\left(v_{i(t)}-v_{j(t)}\right)\right. \\
& \left.+k_{a}\left(a_{i(t)}-a_{j(t)}\right)\right], \quad i \in \mathscr{V}_{f},
\end{aligned}\right.
$$

where $k_{a}, k_{v}$ and $k_{x}$ are positive constant control gains. Note that, for brevity, we have dropped the subscript $(t)$ in (8) for the remainder of this section. System (8) can be given in matrix-vector form by

$$
\left\{\begin{aligned}
\dot{x}_{f}= & v_{f} \\
\dot{v}_{f}= & a_{f} \\
\dot{a}_{f}= & -k_{x}\left[\left(\Omega_{f f} \otimes I_{d}\right) x_{f}+\left(\Omega_{f l} \otimes I_{d}\right) x_{l}^{*}\right] \\
& -k_{v}\left[\left(\Omega_{f f} \otimes I_{d}\right) v_{f}+\left(\Omega_{f l} \otimes I_{d}\right) v_{l}^{*}\right] \\
& -k_{a}\left[\left(\Omega_{f f} \otimes I_{d}\right) a_{f}+\left(\Omega_{f l} \otimes I_{d}\right) a_{l}^{*}\right]
\end{aligned}\right.
$$

where the states $x_{f}, v_{f}, a_{f} \in \mathbb{R}^{d n_{f}}$ respectively denote the position, velocity and acceleration of the followers while $x_{l}^{*}, v_{l}^{*}=\dot{x}_{l}$ and $a_{l}^{*}=\dot{v}_{l}$ have been used to denote those of the leaders respectively. Define the position tracking error as

$$
\delta_{x_{f}}=x_{f}-x_{f}^{*}=x_{f}+\left(\Omega_{f f}^{-1} \Omega_{f l} \otimes I_{d}\right) x_{l}^{*},
$$

where $x_{f}^{*}$ denotes the target positions of the followers. Next, the stability of control law (9) is investigated. Let $\mu_{i}$ denote the $i$ th eigenvalue of $-\Omega_{f f}$.

Theorem 4.1. Assume that Assumptions 1 and 2 hold, and the leaders jerk $\dot{a}_{l}$ is constantly zero, then by choosing the control gains such that $-k_{v} k_{a} \mu_{i}>k_{p}, \forall i$, control law (9) guarantees that the errors in the followers positions $\delta_{x_{f}}$ converge to zero.

Proof. Define the disagreements of the position, velocity and acceleration respectively by $\delta_{x_{f}}=x_{f}+\left(\Omega_{f f}^{-1} \Omega_{f l} \otimes I_{d}\right) x_{l}^{*}, \delta_{v_{f}}=$ $v_{f}+\left(\Omega_{f f}^{-1} \Omega_{f l} \otimes I_{d}\right) v_{l}^{*}$ and $\delta_{a_{f}}=a_{f}+\left(\Omega_{f f}^{-1} \Omega_{f l} \otimes I_{d}\right) a_{l}^{*}$ so that (9) can be re-written as functions of their disagreements as

$$
\begin{aligned}
{\left[\begin{array}{c}
\dot{\delta}_{x_{f}} \\
\dot{\delta}_{v_{f}} \\
\dot{\delta}_{a_{f}}
\end{array}\right]=} & (\underbrace{\left[\begin{array}{ccc}
\mathbf{0}_{n_{f} \times n_{f}} & I_{n_{f}} & \mathbf{0}_{n_{f} \times n_{f}} \\
\mathbf{0}_{n_{f} \times n_{f}} & \mathbf{0}_{n_{f} \times n_{f}} & I_{n_{f}} \\
-k_{p} \Omega_{f f} & -k_{v} \Omega_{f f} & -k_{a} \Omega_{f f}
\end{array}\right]}_{F_{1}} \otimes I_{d})\left[\begin{array}{c}
\delta_{x_{f}} \\
\delta_{v_{f}} \\
\delta_{a_{f}}
\end{array}\right] \\
& +\left(\left[\begin{array}{c}
\mathbf{0}_{n_{f} \times n_{f}} \\
\mathbf{0}_{n_{f} \times n_{f}} \\
\Omega_{f f}^{-1} \Omega_{f l}
\end{array}\right] \otimes I_{d}\right) \dot{a}_{l}^{*} .
\end{aligned}
$$

Note that $\dot{a}_{l}^{*}=0$ for this study. The system matrix of (10) has the characteristic polynomial given by

$$
\begin{aligned}
& \operatorname{det}\left(\left[s I_{3 n_{f}}-F_{1}\right] \otimes I_{d}\right)= \\
& \operatorname{det}\left(\left[\begin{array}{ccc}
s I_{n_{f}} & -I_{n_{f}} & \mathbf{0}_{n_{f} \times n_{f}} \\
\mathbf{0}_{n_{f} \times n_{f}} & s I_{n_{f}} & -I_{n_{f}} \\
k_{p} \Omega_{f f} & k_{v} \Omega_{f f} & \left(s I_{n_{f}}+k_{a} \Omega_{f f}\right)
\end{array}\right] \otimes I_{d}\right) .
\end{aligned}
$$


Note that $\mu_{i}$ denotes the $i$ th eigenvalue of $-\Omega_{f f}$. Thus, $\operatorname{det}\left(s I_{n}+\right.$ $\left.\Omega_{f f}\right)=\prod_{i=1}^{n}\left(s-\mu_{i}\right)$. This implies that

$$
\begin{aligned}
\operatorname{det}\left(\left[s I_{3 n_{f}}-F_{1}\right] \otimes I_{d}\right) & =\operatorname{det}\left(s I_{3 n f}-F_{1}\right) \\
& =\Pi_{i=1}^{n}\left[s^{3}-k_{a} \mu_{i} s^{2}-k_{v} \mu_{i} s-k_{p} \mu_{i}\right] .
\end{aligned}
$$

Therefore, the eigenvalues satisfy

$$
s^{3}-k_{a} \mu_{i} s^{2}-k_{v} \mu_{i} s-k_{p} \mu_{i}=0 .
$$

Since $k_{a}, k_{v}, k_{x}>0$ and $-\mu_{i}>0, \forall_{i}$, all errors converge to zero if and only if $-k_{v} k_{a} \mu_{i}>k_{p}, \forall_{i}$.

Remark 4.2. The choice of $-k_{v} k_{a} \mu_{\max }>k_{p}$ satisfies the convergence requirement $-k_{v} k_{a} \mu_{i}>k_{p}$ for all followers. Note that $\mu_{\max }$ has been used to denote the greatest eigenvalue of $-\Omega_{f f}$, i.e., the greatest $\mu_{i}$.

Remark 4.3. Note that the case of time-varying jeck can be studied using the protocol

$$
\left\{\begin{aligned}
\dot{x}_{i(t)}= & v_{i(t)}, \\
\dot{v}_{i(t)}= & a_{i(t)}, \\
\dot{a}_{i(t)=} & -\frac{1}{\gamma} \sum_{j \in \mathscr{N}_{i}} w_{i j}\left[k_{x}\left(x_{i(t)}-x_{j(t)}\right)+k_{v}\left(v_{i(t)}-v_{j(t)}\right)\right. \\
& \left.+k_{a}\left(a_{i(t)}-a_{j(t)}\right)-\dot{a}_{j}\right], \quad i \in \mathscr{V}_{f},
\end{aligned}\right.
$$

where $\gamma=\sum_{j \in \mathscr{N}_{i}}$. Further stability analysis is not included in this paper because of page limitation.

\section{Affine Formation Control Law for Sampled-data Coordination of Multi-agent Systems Described by Triple-Integrator Agent Dynamics}

Consider the MAS coordination case where the agents are described with triple-integrator dynamics modelled in continuoustime by

$$
\dot{x}_{i(t)}=v_{i(t)}, \quad \dot{v}_{i(t)}=a_{i(t)}, \quad \dot{a}_{i(t)}=u_{i(t)} .
$$

Assume that each agent has continuous-time dynamics, but communicate (or sense) their neighbours at periodic time intervals and their control inputs are zero-order hold based. Such that, following [23],

$$
u_{i}(t)=u_{i}(k T), \quad k T \leq t<(k+1) T,
$$

where $T, k, u_{i(t)}$, and $u_{i[k]}$ respectively denote sampling period, discrete-time index, control input at time $t$ (continuous-time), and control input at $t=k T$.

Discretization of (12) yields

$$
\left\{\begin{array}{l}
x_{i[k+1]}=x_{i[k]}+T v_{i[k]}+\frac{T^{2}}{2} a_{i[k]}+\frac{T^{3}}{6} u_{i[k]}, \\
v_{i[k+1]}=v_{i[k]}+T a_{i[k]}+\frac{T^{2}}{2} u_{i[k]}, \\
a_{i[k+1]}=a_{i[k]}+T u_{i[k]}, \quad i \in \mathscr{V}_{f},
\end{array}\right.
$$

where $a_{i}, v_{i}$ and $x_{i}$ have respectively been used to denote the acceleration, velocity and position of agent $i$. Next, we study the sampled-data case where the jerk of leaders are constantly zero. Consider the protocol,

$$
\begin{aligned}
u_{i[k]}= & -\sum_{j \in \mathscr{N}_{i}} w_{i j}\left[k_{x}\left(x_{i[k]}-x_{j[k]}\right)+k_{v}\left(v_{i[k]}-v_{j[k]}\right)\right. \\
& \left.+k_{a}\left(a_{i[k]}-a_{j[k]}\right)\right], \quad i \in \mathscr{V}_{f},
\end{aligned}
$$

which is given in closed-loop form by

$$
\begin{aligned}
u_{f}= & -k_{x}\left[\left(\Omega_{f f} \otimes I_{d}\right) x_{f}+\left(\Omega_{f l} \otimes I_{d}\right) x_{l}^{*}\right] \\
& -k_{v}\left[\left(\Omega_{f f} \otimes I_{d}\right) v_{f}+\left(\Omega_{f l} \otimes I_{d}\right) v_{l}^{*}\right] \\
& -k_{a}\left[\left(\Omega_{f f} \otimes I_{d}\right) a_{f}+\left(\Omega_{f l} \otimes I_{d}\right) a_{l}^{*}\right] .
\end{aligned}
$$

Using (14), (13) can be re-written in matrix form as

$$
\begin{aligned}
& {\left[\begin{array}{c}
x_{f[k+1]} \\
v_{f[k+1]} \\
a_{f[k+1]}
\end{array}\right]=} \\
& (\underbrace{\left[\begin{array}{ccc}
\left(I_{n f}-\frac{T^{3}}{6} k_{x} \Omega_{f f}\right) & \left(T I_{n_{f}}-\frac{T^{3}}{6} k_{v} \Omega_{f f}\right) & \left(\frac{T^{2}}{2} I_{n f}-\frac{T^{3}}{6} k_{a} \Omega_{f f}\right) \\
-\frac{T^{2}}{2} k_{x} \Omega_{f f} & \left(I_{n f}-\frac{T^{2}}{2} k_{v} \Omega_{f f}\right) & \left(T I_{n_{f}}-\frac{T^{2}}{2} k_{a} \Omega_{f f}\right) \\
-T k_{x} \Omega_{f f} & -T k_{v} \Omega_{f f} & \left(I_{n f}-T k_{a} \Omega_{f f}\right)
\end{array}\right]}_{F_{2}} \otimes I_{d}) \\
& \times\left[\begin{array}{c}
x_{f[k]} \\
v_{f[k]} \\
a_{f[k]}
\end{array}\right] \\
& +\left(\left[\begin{array}{c}
-\frac{T^{3}}{6} k_{x} \Omega_{f l}-\frac{T^{3}}{6} k_{v} \Omega_{f l}-\frac{T^{3}}{6} k_{a} \Omega_{f l} \\
-\frac{T^{2}}{2} k_{x} \Omega_{f l}-\frac{T^{2}}{2} k_{v} \Omega_{f l}-\frac{T^{2}}{2} k_{a} \Omega_{f l} \\
-T k_{x} \Omega_{f l}-T k_{v} \Omega_{f l}-T k_{a} \Omega_{f l}
\end{array}\right] \otimes I_{d}\right)\left[\begin{array}{l}
x_{l[k]}^{*} \\
v_{l[k]}^{*} \\
a_{l[k]}^{*}
\end{array}\right]
\end{aligned}
$$

where $v_{l}^{*} \in \mathbb{R}^{d n_{l}}$ and $v_{f} \in \mathbb{R}^{d n_{f}}$ is used to respectively denote the velocities of the leaders and followers. Define the position tracking error as

$$
\delta_{f[k]}=x_{f[k]}-x_{f[k]}^{*}=x_{f[k]}+\left(\Omega_{f f}^{-1} \Omega_{f l} \otimes I_{d}\right) x_{[[k]}^{*},
$$

where $x_{f[k]}^{*}$ denotes the target positions of the followers. Next, we analyse the stability of control law (15).

Theorem 5.1. Assume that Assumptions 1 and 2 hold, and the leaders jerk is constantly zero, by choosing $k_{x}, k_{a}, k_{v}$ and $T$ such that $\left(2 k_{v}-T k_{x}\right)>0$ and $k_{v}\left(3 T k_{v}-T^{2} k_{x}-6 k_{a}\right) \mu_{i}-6 k_{x}>0$, the tracking error $\delta_{x_{f}[k]}$ of all followers is guaranteed to stabilize to the origin by control law (15).

Proof. Let the state errors (or disagreements) of the followers be defined by

$$
\begin{aligned}
{\left[\delta_{x_{f}[k]}^{T}, \delta_{v_{f}[k]}^{T}, \delta_{a_{f}[k]}^{T}\right]^{T}=} & {\left[x_{f[k]}^{T}, v_{f[k]}^{T}, a_{f[k]}^{T}\right]^{T} } \\
& -\left[x_{f[k]}^{* T}, v_{f[k]}^{* T}, a_{f[k]}^{* T}\right]^{T},
\end{aligned}
$$

where $a_{f}^{*}, v_{f}^{*}, x_{f}^{*} \in \mathbb{R}^{d n_{f}}$ respectively denote the target accelerations, velocities and positions of the followers. By taking (16) and control law (15) into consideration, the system matrix of the error dynamics is defined by 


$$
\underbrace{\left[\begin{array}{ccc}
\left(I_{n f}-\frac{T^{3}}{6} k_{x} \Omega_{f f}\right) & \left(T I_{n_{f}}-\frac{T^{3}}{6} k_{v} \Omega_{f f}\right) & \left(\frac{T^{2}}{2} I_{n f}-\frac{T^{3}}{6} k_{a} \Omega_{f f}\right) \\
-\frac{T^{2}}{2} k_{x} \Omega_{f f} & \left(I_{n f}-\frac{T^{2}}{2} k_{v} \Omega_{f f}\right) & \left(T I_{n_{f}}-\frac{T^{2}}{2} k_{a} \Omega_{f f}\right) \\
-T k_{x} \Omega_{f f} & -T k_{v} \Omega_{f f} & \left(I_{n f}-T k_{a} \Omega_{f f}\right)
\end{array}\right]}_{F_{2}} \otimes I_{d} .
$$

The characteristic polynomial of $F_{2} \otimes I_{d}$ in (17) is given by $\operatorname{det}\left(\left[s I_{3 n f}-F_{2}\right] \otimes I_{d}\right)$, which equals $\operatorname{det}\left(s I_{3 n f}-F_{2}\right)$. Note that $\mu_{i}$ is used to denote the $i$ th eigenvalue of $-\Omega_{f f}$. Thus, $\operatorname{det}\left(s I_{n}+\right.$ $\left.\Omega_{f f}\right)=\Pi_{i=1}^{n}\left(s-\mu_{i}\right)$. It implies that (17) satisfies

$$
\begin{aligned}
& s^{3}-\left(\frac{T^{3}}{6} k_{x} \mu_{i}+\frac{T^{2}}{2} k_{v} \mu_{i}+T k_{a} \mu_{i}+3\right) s^{2} \\
& +\left(-\frac{2 T^{3}}{3} k_{x} \mu_{i}+2 T k_{a} \mu_{i}+3\right) s \\
& -\left(\frac{T^{3}}{6} k_{x} \mu_{i}-\frac{T^{2}}{2} k_{v} \mu_{i}+T k_{a} \mu_{i}+1\right)=0 .
\end{aligned}
$$

Using the bi-linear transform $s=\frac{t+1}{t-1}$, for (18), the expression

$$
\begin{aligned}
& -3 T^{3} k_{x} \mu_{i} t^{3}+\left(3 T^{3} k_{x} \mu_{i}-6 T^{2} k_{v} \mu_{i}\right) t^{2} \\
& +\left(T^{3} k_{x} \mu_{i}+6 T^{2} k_{v} \mu_{i}-12 T k_{a} \mu_{i}\right) t \\
& +\left(-T^{3} k_{x} \mu_{i}+12 T k_{a} \mu_{i}+24\right)=0
\end{aligned}
$$

is obtained. Thus, a sufficient condition for the stability of (15) is for all the roots of (19) to be on the left half plane. Note that the entire roots of (18) fall within a unit circle if the entire roots of (19) are situated in the open left half plane. Note that $-3 T^{3} k_{x} \mu_{i}>0$, because $\mu_{i}$ is always negative. Thus, guaranteeing that

$$
\begin{aligned}
& 3 T^{3} k_{x} \mu_{i}-6 T^{2} k_{v} \mu_{i}>0, \quad \text { and } \\
& \left(3 T^{3} k_{x} \mu_{i}-6 T^{2} k_{v} \mu_{i}\right)\left(T^{3} k_{x} \mu_{i}+6 T^{2} k_{\nu} \mu_{i}-12 T k_{a} \mu_{i}\right) \\
& +3 T^{3} k_{x} \mu_{i}\left(-T^{3} k_{x} \mu_{i}+12 T k_{a} \mu_{i}+24\right)>0,
\end{aligned}
$$

guarantees that the roots of (18) are within the unit circle. By further algebraic simplification, these requirements are respectively reduced to $\left(2 k_{v}-T k_{x}\right)>0$ and $k_{v}\left(3 T k_{v}-T^{2} k_{x}-6 k_{a}\right) \mu_{i}-6 k_{x}>$ 0 .

\section{Implementation}

The first step in the implementation of the proposed algorithms is the design of a reference formation that satisfies Assumptions 1 and 2 . That is, the reference formation should be both generically universally rigid and have at least $d+1$ nodes, that span the $\mathbb{R}^{d}$ space affinely, to be selected as leaders. The next step is to compute the stress matrix $\Omega$. Equation (3) is used to compute the stress matrix in this study.

The simulations were done in obstacle avoidance scenarios. The acceleration varied slowly at some points, however, the control algorithms are still effective in these cases. We now present the results of our four implemention cases grouped into two scenarios. Note that, the agents' connections in the framework (e.g. in Fig.6) are denoted with straight lines and $(2,0)$ is used to denote that the agent's position on the $x$ - and $y$-axis are respectively 2 and 0.

\subsection{Scenario One}

Consider a five-agent MAS where each agent is modelled as triple-integrators. Assume that the agents are denoted by $i=$ $1,2, \ldots, 5$. Let Fig. 1 denote the communication graph of the agents.

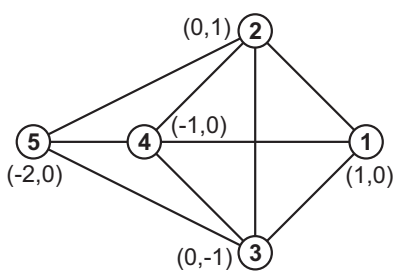

Fig. 1. Framework showing agent communication along with their 2dimensional reference positions.

Agents 1 to 3 are selected as leaders while the rest are followers. The stress matrix of the graph is given by

$$
\Omega=\left[\begin{array}{ll}
\Omega_{l l} & \Omega_{l f} \\
\Omega_{f l} & \Omega_{f f}
\end{array}\right]
$$

where

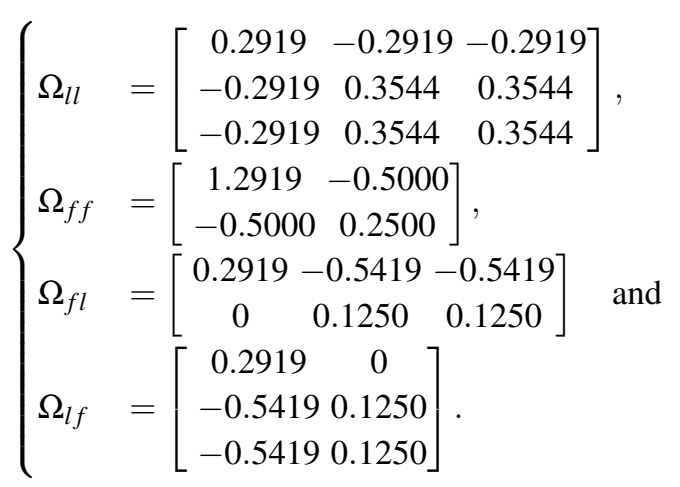

The largest eigenvalue of $-\Omega_{f f}, \mu_{\max }=-0.049$. The leaders' initial positions are their respective nominal positions while the followers are initialized to the following positions: $P_{4}(-3,1)$ and $P_{5}(-1,2)$, where $P_{5}(-1,2)$ implies that the position of agent 5 is $(-1,2)$. We now present the results of two simulation studies where the leaders paths are generated in advance. 


\subsubsection{Continuous-time Inter-Agent Communication Case}

This study considers the case where inter-agent communication occurs continuously in time. We use control law (9) in this study. The control parameters are chosen to satisfy the requirement of Theorem 4.1 , i.e., $-k_{v} k_{a} \mu_{i}>k_{p}, \forall i$. Here, we consider $-k_{v} k_{a} \mu_{\max }>k_{p}$, which is the lower bound. From (20), $\mu_{\max }=$ -0.049 for $-\Omega_{f f}$. It is easy to verify that the choice of the control gains $k_{x}=0.8, k_{v}=8$ and $k_{a}=10$ with $\mu_{\max }=-0.049$ satisfy the requirement of Theorem $4.1\left(-k_{v} k_{a} \mu_{i}>k_{p}, \forall i\right)$. These parameters are used in the simulation.

In the simulation study, the velocities and accelerations of the leaders are estimated using discrete differentiators (zeroorder hold based). Note that the trajectories of the leaders is assumed to be piecewise continuous and differentiable.

The simulation result showing the agents' positions is presented in Fig. 2. It shows the formation maneuver around obstacles on its path. The trajectories of the agents' velocities and accelerations are presented in Fig. 11 and the tracking errors of the followers' positions are presented in Fig. 3.

\subsubsection{Sampled-data Inter-Agent Communication Case}

This study considers the case where inter-agent communication occurs in periodic time intervals. We use control law (15) in this study. The control parameters are chosen to satisfy the requirements of Theorem 5.1. Denote the least eigenvalue of $-\Omega_{f f}$ by $\mu_{\text {min }}$. It can be verified from (20), that $\mu_{\text {max }}=-0.049$ and $\mu_{\text {min }}=-1.493$ in this case. It is easy to verify that the choices of $T=0.1, k_{x}=0.8, k_{v}=8$ and $k_{a}=8$ satisfy the requirements of Theorem 5.1, i.e., $\left(2 k_{v}-T k_{x}\right)>0$ and $k_{v}\left(3 T k_{v}-T^{2} k_{x}-\right.$ $\left.6 k_{a}\right) \mu_{i}-6 k_{x}>0, \forall i$. Note that both $\mu_{\max }$ and $\mu_{\text {min }}$ are used because they form the boundary.

The simulation result showing the agents' positions is presented in Fig. 4. It shows the formation maneuver around obstacles on its path. The trajectories of the agents' velocities and accelerations are presented in Fig. 12 and the tracking errors of the followers' positions are shown in Fig. 5.

\subsection{Scenario Two}

Consider a seven-agent MAS where each agent is modelled as triple-integrators. Assume that the agents are denoted by $i=1,2, \ldots, 7$. Let Fig. 6 denote the communication graph of the agents.

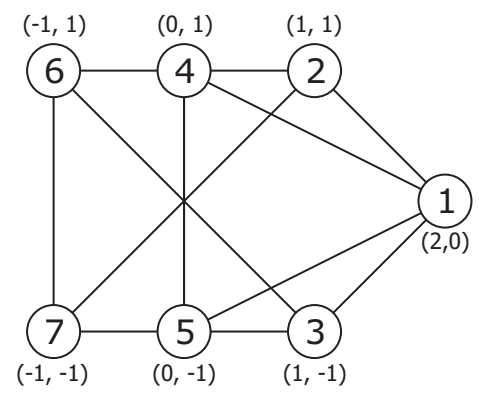

Fig. 6. Framework showing agent communication along with their 2dimensional reference positions.

Agents 1 to 3 are selected as leaders while the rest are followers. The stress matrix of the graph is given by [14].

$$
\Omega=\left[\begin{array}{ll}
\Omega_{l l} & \Omega_{l f} \\
\Omega_{f l} & \Omega_{f f}
\end{array}\right]
$$

where

$$
\begin{aligned}
\Omega_{l l}= & {\left[\begin{array}{ccc}
0.2742 & -0.2741 & -0.2741 \\
-0.2741 & 0.6853 & 0 \\
-0.2741 & 0 & 0.6853
\end{array}\right], } \\
\Omega_{f f}= & {\left[\begin{array}{cccc}
0.7538 & -0.0685 & -0.2741 & 0 \\
-0.0685 & 0.7538 & 0 & -0.2741 \\
-0.2741 & 0 & 0.2741 & -0.1370 \\
0 & -0.2741 & -0.1370 & 0.2741
\end{array}\right], } \\
\Omega_{f l}= & {\left[\begin{array}{ccc}
0.1370 & -0.5482 & 0 \\
0.1370 & 0 & -0.5482 \\
0 & 0 & 0.1370 \\
0 & 0.1370 & 0
\end{array}\right] \text { and } } \\
\Omega_{l f}= & {\left[\begin{array}{ccc}
0.1370 & 0 & 0 \\
0 & 0 & 0.1370 \\
-0.5482 & 0.1370 & 0
\end{array}\right] }
\end{aligned}
$$

The largest eigenvalue of $-\Omega_{f f}, \mu_{\max }=-0.024$. The leaders' initial positions are their respective nominal positions while the followers are initialized to the following positions: $P_{4}(0,2), P_{5}(0,-2), P_{6}(-1,3)$ and $P_{7}(-1,3)$, where $P_{5}(0,-2)$ implies that the position of agent 5 is $(0,-2)$. We now present the results of two simulation studies where the leaders paths are generated in advance.

\subsubsection{Continuous-time Inter-Agent Communication Case}

This study considers the case where inter-agent communication occurs continuously in time. We use control law (9) in this study. Note that in this case $\mu_{\max }=-0.024$. The control gains $k_{x}, k_{v}$ and $k_{a}$ are respectively chosen to be $0.8,8$ and 9 . It is easy to verify that this satisfies the requirement of Theorem 4.1, i.e., $-k_{v} k_{a} \mu_{i}>k_{p}, \forall i$. These parameters are used in the simulation.

In the simulation study, the velocities and accelerations of the leaders are estimated using discrete differentiators (zeroorder hold based). Note that the trajectories of the leaders is assumed to be piecewise continuous and differentiable.

The simulation result showing the agents' positions is presented in Fig. 7. It shows the formation maneuver around obstacles on its path. The trajectories of the agents' velocities and accelerations are presented in Fig. 13 and the tracking errors of the followers' positions are presented in Fig. 8. 


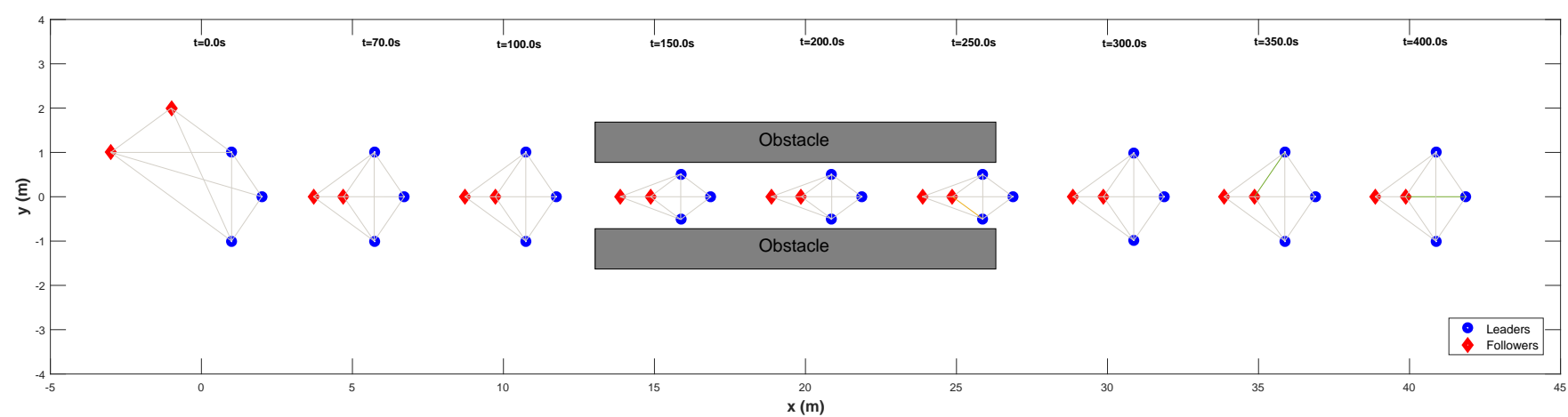

Fig. 2. Simulation illustrating the agents' positions in the continuous-time case of scenario one, based on control law (9)

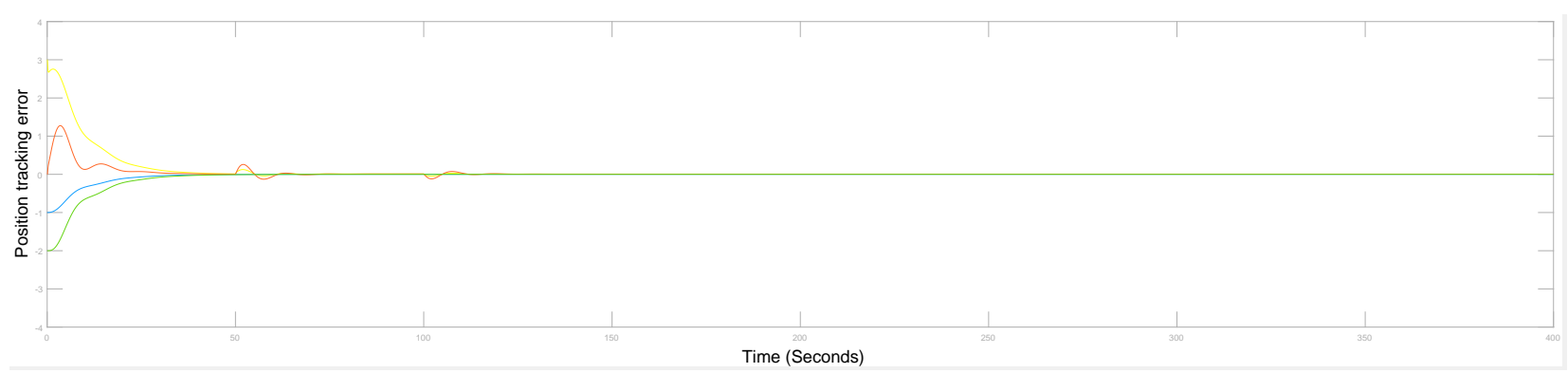

Fig. 3. Simulation illustrating the positions error dynamics for the continuous-time case of scenario one

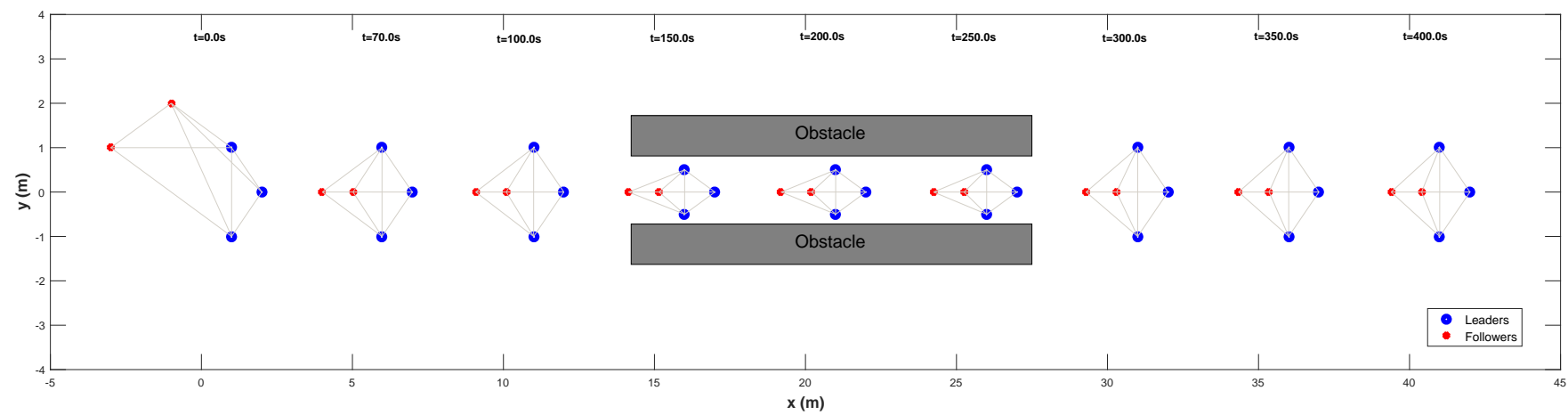

Fig. 4. Simulation illustrating the agents' positions in the sampled-data case of scenario one, based on control law (15)

\subsubsection{Sampled-data Inter-Agent Communication Case}

This study considers the case where inter-agent communication occurs in periodic time intervals. We use control law (15) in this study. Denote the least eigenvalue of $-\Omega_{f f}$ by $\mu_{\text {min }}$. It can be verified from (21) that $\mu_{\max }=-0.024$ and $\mu_{\text {min }}=-0.96$ in this case. Thus, the choices of $T=0.1, k_{x}=0.8, k_{v}=8$ and $k_{a}=8$ can be verified to satisfy the requirements of Theorem 5.1, i.e., $\left(2 k_{v}-T k_{x}\right)>0$ and $k_{v}\left(3 T k_{v}-T^{2} k_{x}-6 k_{a}\right) \mu_{i}-6 k_{x}>0, \forall i$. Note that both $\mu_{\text {max }}$ and $\mu_{\text {min }}$ are used because they form the boundary.

The simulation result showing the agents' positions is pre- sented in Fig. 9. It shows the formation maneuver around obstacles on its path. The trajectories of the agents' velocities and accelerations are presented in Fig. 14 and the tracking errors of the followers' positions are presented in Fig. 10.

\section{Conclusion}

This study investigates the affine formation control of MASs with triple-integrator agent dynamics in both sampled-data and continuous-time settings. Algorithms, based on stress matrix, are proposed to accomplish formation control in each setting. Sufficient conditions on the sampling intervals and control gains for 


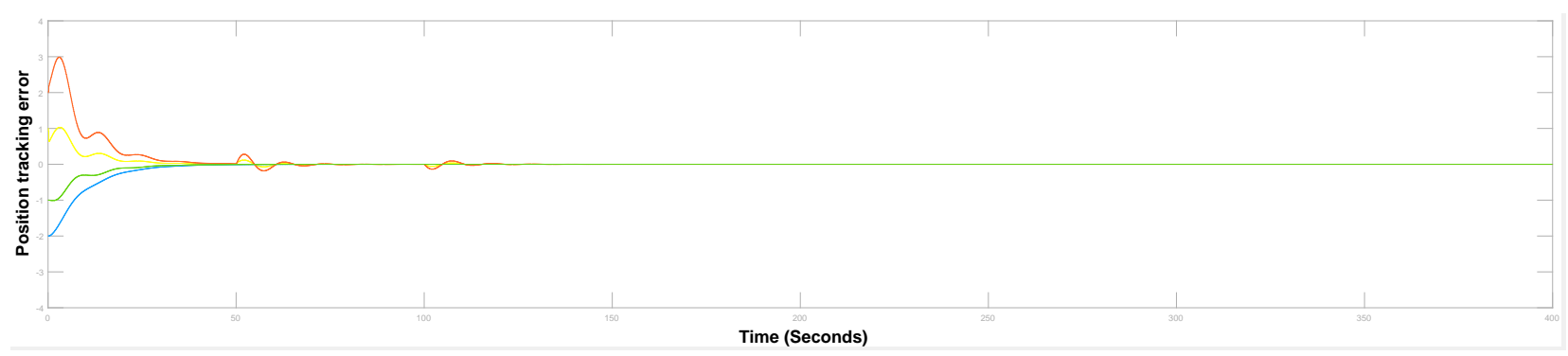

Fig. 5. Simulation illustrating the positions error dynamics for the sampled-data case of scenario one

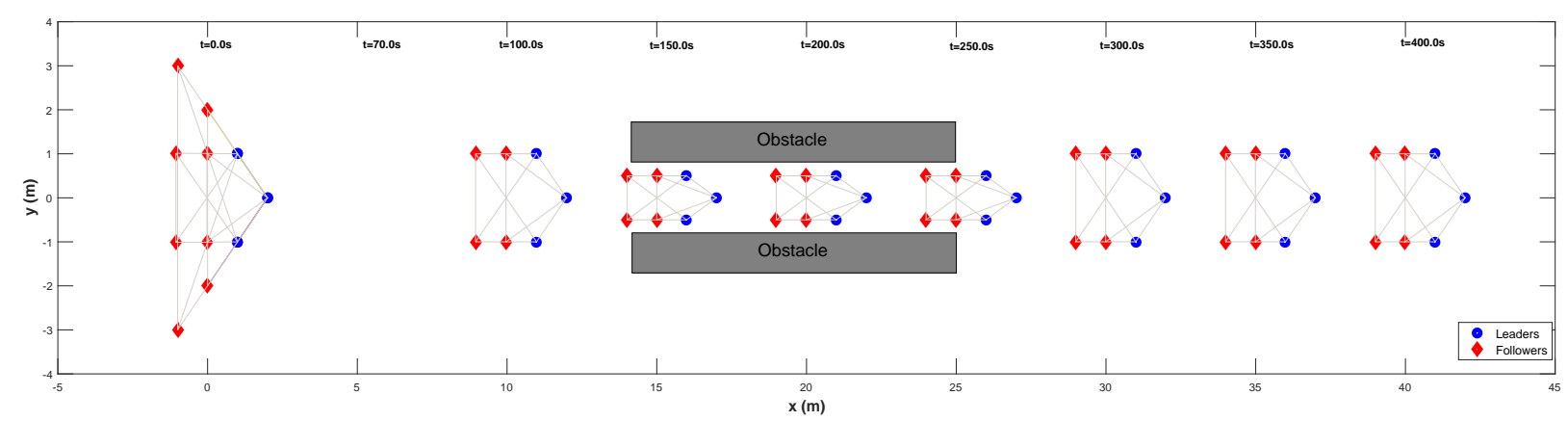

Fig. 7. Simulation illustrating the agents' positions in the continuous-time case of scenario two, based on control law (9).

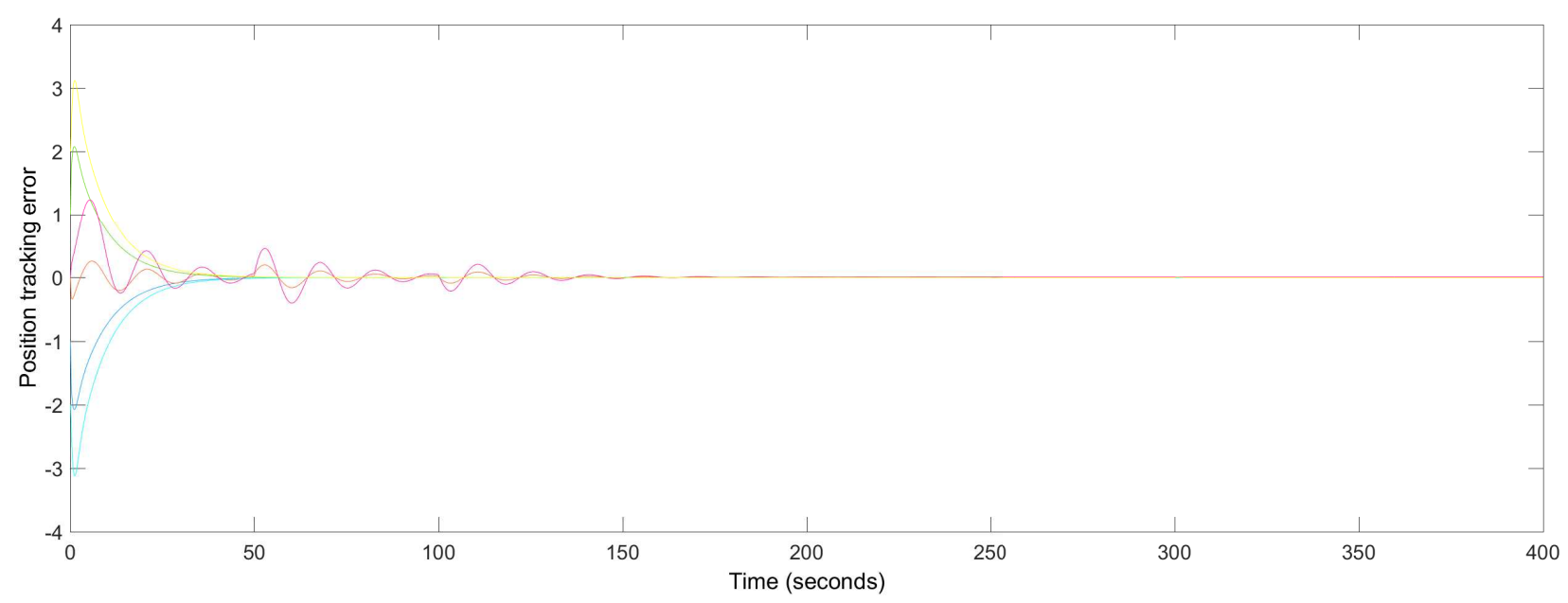

Fig. 8. Simulation illustrating the positions error dynamics for the continuous-time case of scenario two.

the overall stability of the formation are presented. Implementations were carried out for four cases. The results obtained in all four cases are in agreement with the proposed algorithms. The proposed control algorithms are capable of tracking timevarying transformations that are the affine transform of the nominal formation if the jerk of the agents is zero. An ongoing study is on extending the scheme to the general linear multi-agent system case.

\section{References}

[1] C. Wang, H. Tnunay, Z. Zuo, B. Lennox and Z. Ding, Fixed-time formation control of multi-robot systems: Design and experiments, IEEE Transactions on Industrial Electronics (To be published).

[2] W. Ren, R. W. Beard and E. M. Atkins, Information consensus in multivehicle cooperative control, IEEE Control Systems 27(2) (2007) 71-82.

[3] S. Zhao and D. Zelazo, Translational and scaling forma- 
March 19, $2019 \quad$ 11:40 manuscript

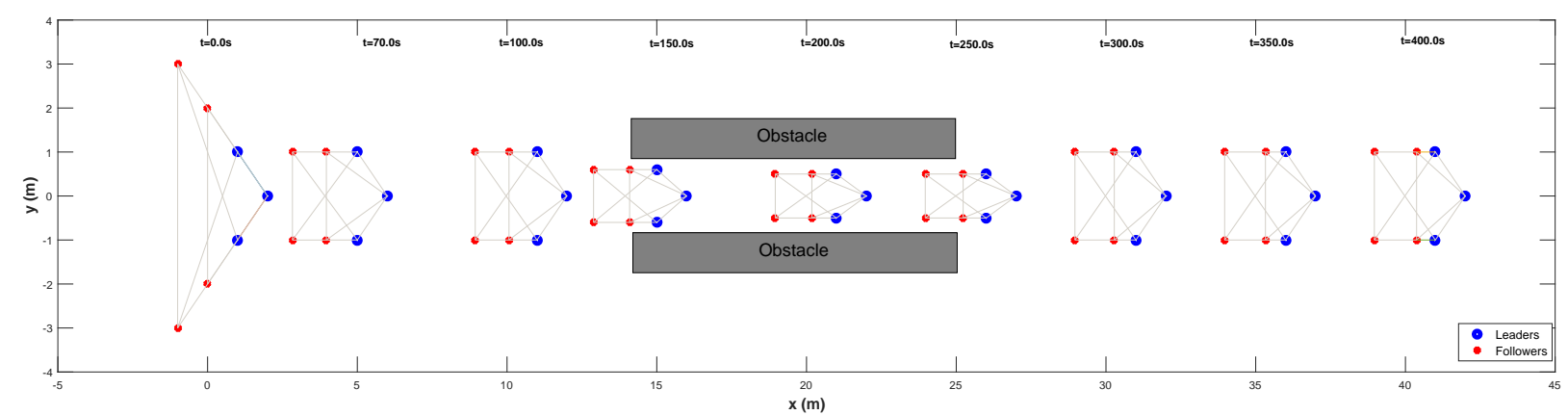

Fig. 9. Simulation illustrating the agents' positions in the sampled-data case of scenario two, based on control law (15).

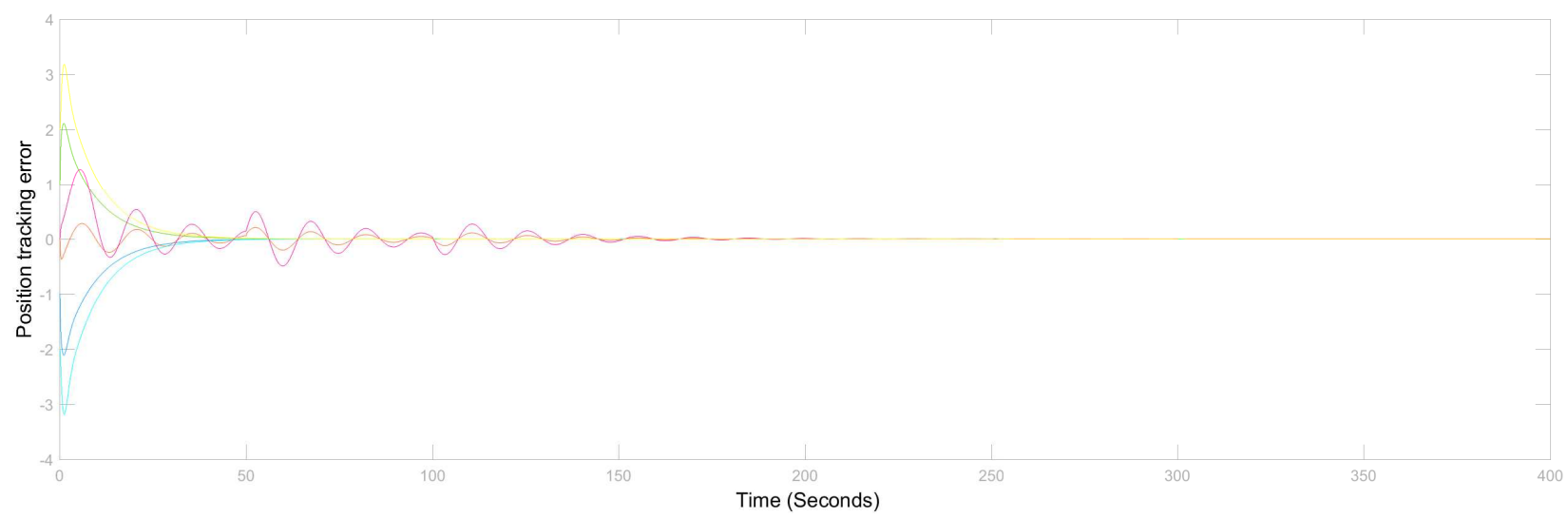

Fig. 10. Simulation illustrating the positions error dynamics for the sampled-data case of scenario two.
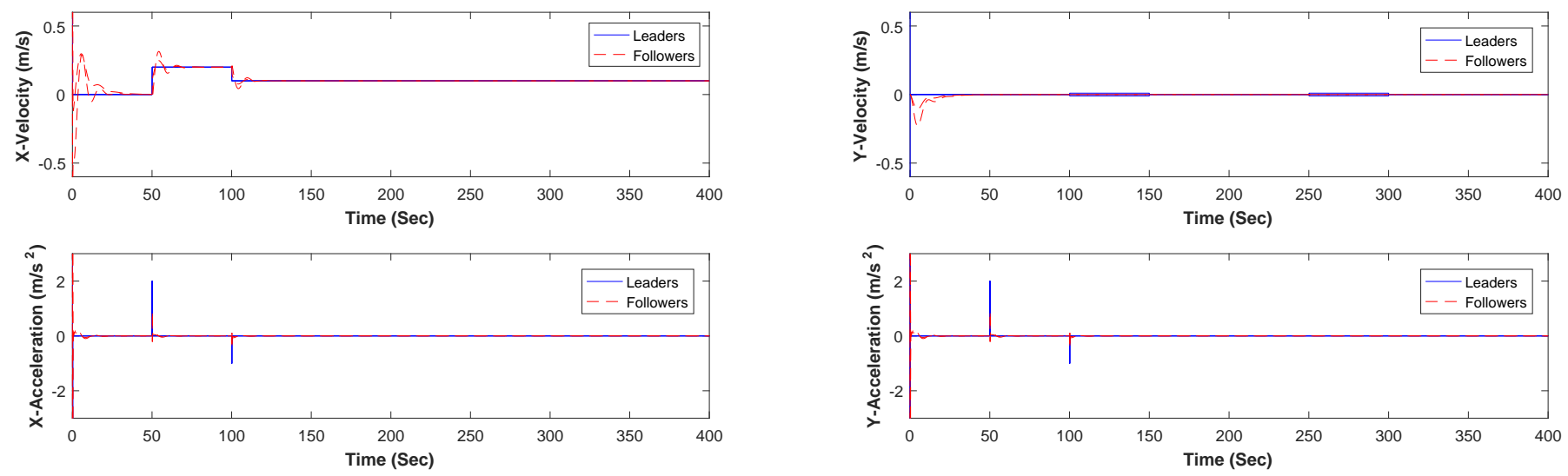

Fig. 11. Simulation depicting the agents' velocity and acceleration trajectories for the continuous-time case of Scenario one.

tion maneuver control via a bearing-based approach, IEEE Transactions on Control of Network Systems 4(3) (2017) 429-438.

[4] K. Oh, M. Park and H. Ahn, A survey of multi-agent formation control, Automatica 53 (2015) 424 - 440.

[5] S. Zhao and D. Zelazo, Bearing rigidity and almost global bearing-only formation stabilization, IEEE Transactions on Automatic Control 61(5) (2016) 1255-1268.

[6] W. Ren, Multi-vehicle consensus with a time-varying reference state, Systems and Control Letters 56(7) (2007) 474 -483 .

[7] W. Ren, On consensus algorithms for double-integrator 

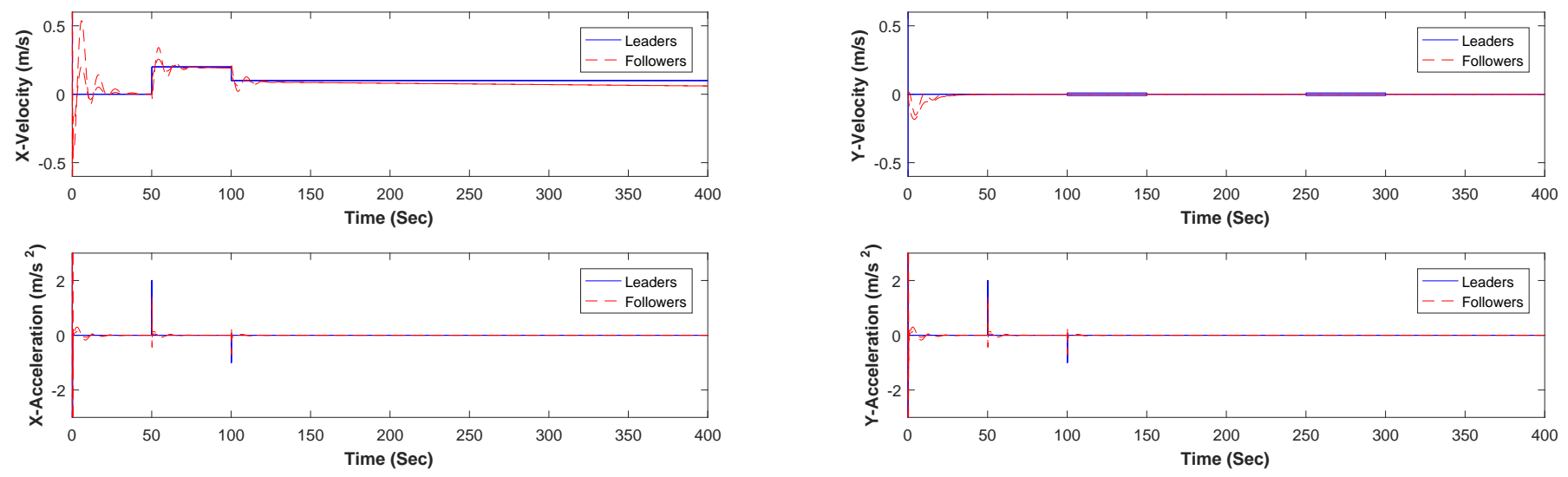

Fig. 12. Simulation depicting the agents' velocity and acceleration trajectories for the sampled-data case of Scenario one.
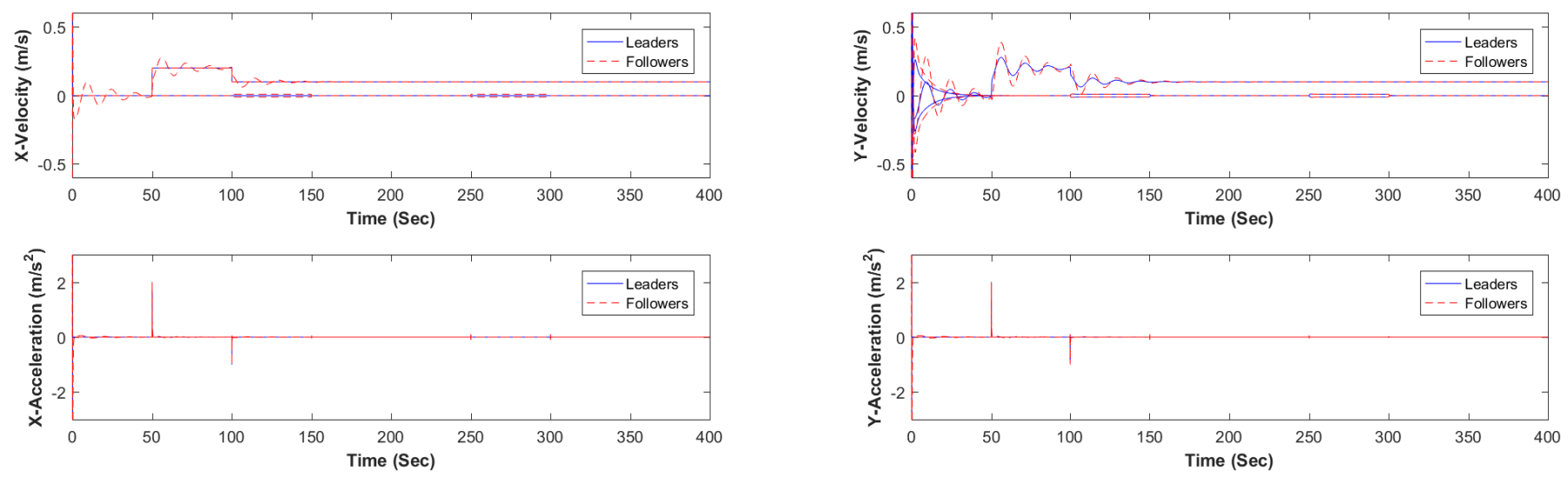

Fig. 13. Simulation depicting the agents' velocity and acceleration trajectories for the continuous-time case of Scenario two.
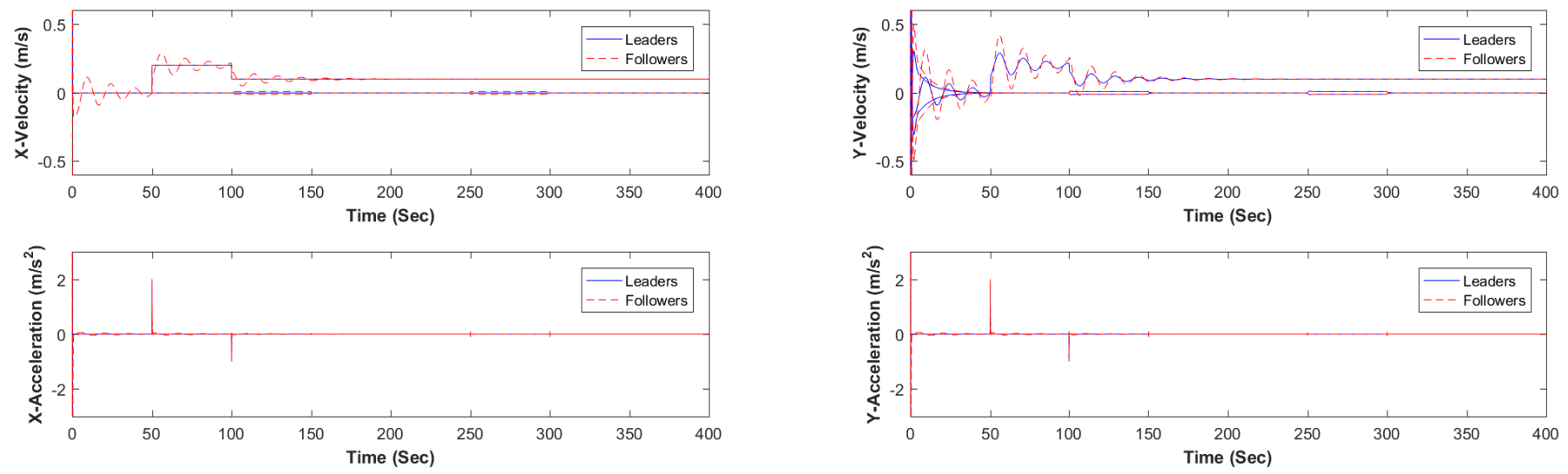

Fig. 14. Simulation depicting the agents' velocity and acceleration trajectories for the sampled-data case of Scenario two. 
[8] H. G. de Marina, B. Jayawardhana and M. Cao, Distributed rotational and translational maneuvering of rigid formations and their applications, IEEE Transactions on Robotics 32(3) (2016) 684-697.

[9] Z. Sun, M. Park, B. Anderson and H. Ahn, Distributed stabilization control of rigid formations with prescribed orientation, Automatica 78 (2017) 250 - 257.

[10] Z. Lin, L. Wang, Z. Han and M. Fu, Distributed formation control of multi-agent systems using complex laplacian, IEEE Transactions on Automatic Control 59(7) (2014) 1765-1777.

[11] Z. Han, L. Wang, Z. Lin and R. Zheng, Formation control with size scaling via a complex laplacian-based approach, IEEE Transactions on Cybernetics 46(10) (2016) 23482359.

[12] Z. Lin, L. Wang, Z. Chen, M. Fu and Z. Han, Necessary and sufficient graphical conditions for affine formation control, IEEE Transactions on Automatic Control 61(10) (2016) 2877-2891.

[13] Q. Yang, M. Cao, Z. Sun, H. Fang and J. Chen, Formation scaling control using the stress matrix, 2017 IEEE 56th Annual Conference on Decision and Control (CDC), (Dec 2017), pp. 3449-3454.

[14] S. Zhao, Affine formation maneuver control of multi-agent systems, IEEE Transactions on Automatic Control (To be published).

[15] M. Bernardo, A. Salvi, S. Santini and A. S. Valente, Thirdorder consensus in vehicles platoon with heterogeneous time-varying delays, 12th IFAC Workshop onTime Delay
Systems TDS 2015 (June 2015) $358-363$.

[16] J. Liu, B. An and H. Wu, Consensus of third-order multiagent systems with communication delay, 2018 Chinese Control And Decision Conference (CCDC), (June 2018), pp. 1428-1432.

[17] W. Hou, M. Fu and H. Zhang, Distributed consensus of third-order multi-agent systems with communication delay, Asian Journal of Control 20(2) (2018) 956-961.

[18] R. Connelly, Generic global rigidity, Discrete \& Computational Geometry 33(4) (2005) 549-563.

[19] R.

Connelly

and S. D. Guest, Frameworks, tensegrities and symmetry: Understanding stable structures (2015) available online at http://www.math.cornell.edu/web7510/framework.pdf.

[20] S. J. Gortler, A. D. Healy and D. P. Thurston, Characterizing Generic Global Rigidity, ArXiv e-prints (2007).

[21] A. Y. Alfakih, On bar frameworks, stress matrices and semidefinite programming, Mathematical Programming 129(1) (2011) 113-128.

[22] Q. Yang, M. Cao, H. Fang and J. Chen, Constructing universally rigid tensegrity frameworks with application in multi-agent formation control], IEEE Transactions on Automatic Control (To be published).

[23] T. Hayakawat, T. Matsuzawat and S. Harat, Formation control of multi-agent systems with sampled information - relationship between information exchange structure and control performance -, Proceedings of the 45th IEEE Conference on Decision and Control, (Dec 2006), pp. 43334338. 